\title{
Simultaneous detection of water-soluble vitamins using the High Performance Liquid Chromatography (HPLC) - a review
}

\author{
E. E. Abano ${ }^{1,2 *}$, Rosemond Godbless Dadzie ${ }^{2}$ \\ ${ }^{1}$ Agricultural Engineering Department, University of Cape Coast, Cape Coast, Ghana \\ ${ }^{2}$ School of Food and Biological Engineering, Jiangsu University, 301 Xuefu Road, Zhenjiang 212013, China
}

review

\section{Summary}

The water-soluble vitamins (WSV): ascorbic acid (vitamin C), thiamine (B1), riboflavin (B2), niacin (B3), panthothenic acid (B5), pyridoxine, and pyridoxal (B6), folic acid (B9), biotin(B8), and B12 are very essential in the diet of humankind. As a result of ever increasing pressures from both consumers and legal enforcers, to specify accurately nutritive compositions of WSV that are present in food materials, many researchers have attempted to fill this niche through the provision of highly sensitive and rapid high performance liquid chromatography (HPLC) procedures. In view of the health benefits of WSV, a replete of HPLC methods have been developed for simultaneous determination of their contents in nature and fortified food samples, nutritional supplements, as well as blood plasmas. The rate of losses of these vitamins during food processing and analysis, in addition to their transient dynamics, presents complexities in developing a highly sensitive HPLC procedure for their simultaneous separations and assays. This review critically assesses the different HPLC procedures developed by researchers and available in the open literature for simultaneous determination of water-soluble vitamins (WSV) in dried tropical fruits materials. The study revealed that not a single chromatographic run developed by researchers can simultaneously elute all the WSV at a time. However, the HPLC procedures that are capable of determining all the WSV were coupled with electrospray ionization mass spectroscopy (ESI-MS), thus making the set-up expensive.

Keywords: water-soluble vitamins, dried tropical fruits, simultaneous, HPLC

\section{Introduction}

Water-soluble vitamins play a very important role in the diet of mankind mainly due to its reducing and antioxidative properties as well as co-enzymatic functions for the metabolism of other nutrients (Owen, 1996). Its health promoting benefits have created increasing awareness of these vitamins in the food and the pharmaceutical industry. Today, the global market is flooded with water-soluble vitamins fortified foods and nutritional supplements. Keeping in mind their importance, the analysis of food products and pharmaceuticals containing these vitamins assume significance in their accurate assays (Arya et al., 2000). Even though the literature is filled up with different simultaneous analysis of the water-soluble vitamins, the high performance liquid chromatography (HPLC) has assumed wide applications because of its sensitivity, selectivity, and utility-based usability. Nevertheless, efforts continue in the search of better HPLC procedures because of lapses in available methods. Such struggles to quantify simultaneously all the water-soluble vitamins in a single HPLC run have resulted in a large number of procedures. This review critically assesses the available HPLC procedures developed by researchers for the simultaneous determination of water-soluble vitamins in dried foods and pharmaceutical products and their user friendliness in terms of time required to prepare stock and standard solutions, the chromatographic procedures involved, as well as the sensitivity of the method. The review focuses particularly on the wider scope of coverage for all the water soluble vitamins by the developed methods and its suitability for routine analysis. In addition, the review assesses the rapidity of the procedures, and its application to dried tropical fruits.

\section{Chemical structures of vitamins studied}

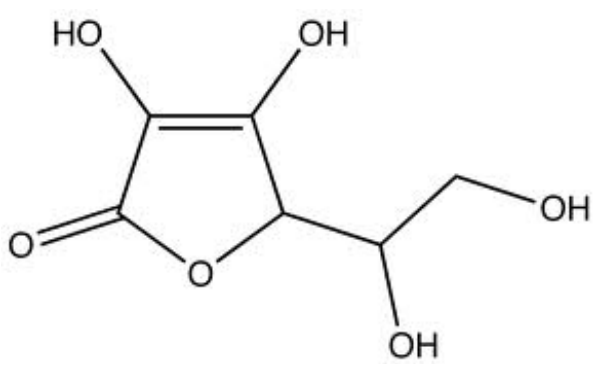


Ascorbic acid (VC)<smiles>Cc1ncc(C[n+]2csc(CCO)c2C)c(N)n1</smiles>

Thiamine (B1)<smiles>Cc1cc2nc3c(=O)[nH]c(=O)nc-3n(CC(O)(O)[C@H](O)[C@H](O)CO)c2cc1C</smiles>

Riboflavin (B2)<smiles>O=C(O)c1cccnc1</smiles>

Niacin (B3)<smiles>CC(C)(CO)C(O)C(=O)NCCC(=O)O</smiles>

Pantothenic acid (B5)<smiles>Cc1ncc(CO)c(CO)c1O</smiles>

Pyridoxal (B6)<smiles>O=C(O)CCC1CSCC1NC(=O)O</smiles>

Biotin (B8)<smiles>Nc1nc2ncc(CNc3ccc(C(=O)N[C@@H](CCC(=O)O)C(=O)O)cc3)nc2c(=O)[nH]1</smiles>

Folic acid (B9)

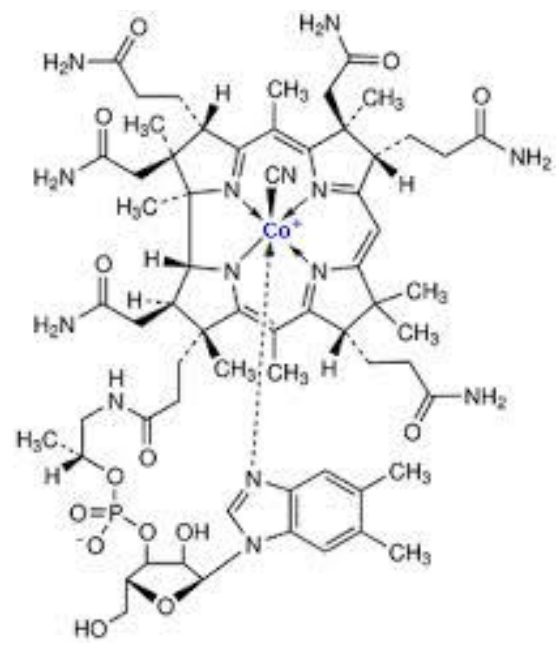

B12

Water-soluble vitamins analyses

Analytical methods in general must be reliable and robust to be useful (Sharpless et al., 2000). The method must be rapid and suitable for routine analysis as well as inexpensive to set up. The analyte must be stable during the analytical process; both the extraction and chromatographic procedures must be reproducible; the results should be accurate and the 
uncertainty should be small; the detector should be selective for the analyses of interest; the signal for each analyte of interest should be free from significant interferences; and the method should be free from matrix effects. Determination of the WSV in foods is often a challenging task due to their instability. Aging, storage conditions, and processing of foods cause WSV loss, which varies widely depending on the type of food and a number of specific factors (e.g. temperature, oxygen, light, moisture, pH) (Leskova et al., 2006). Additionally, for the determination of vitamin concentrations, it is necessary to demonstrate the accuracy of a method by indicating that all of the naturally occurring species of a given vitamin are fully extracted and measured. Scientists at the United States National Institute of Standards and Technology (NIST) measured the challenges that can be encountered in the determination of vitamins and reported that the stability of analyte is an important aspect of assigning values to analytes concentrations in a certified reference material. They argued that certain vitamins and caroteniods may function as antioxidants, which makes them unstable by their very nature, hence may be degraded when exposed to air, light, or heat (NIST, 2014).

\section{Storage of Reference and Test Materials}

Storage of reference material is as important as the preparation and analysis of test samples. For example, it has been reported that vitamin $\mathrm{C}$ is degraded to dehydroascorbic acid and smaller molecules when stored or analysed above $4{ }^{\circ} \mathrm{C}$. Hence, considering the different ambient climatic conditions in the scientific laboratories worldwide and unstable nature of some water-soluble vitamins, an ideal and feasible ambient condition for storage, preparation, and analysis of both reference and sample/test materials should be critical. In view of this, researchers should handle reference and test materials in an environment that allows for accurate separation and determination of the WSV. Studies have shown that storage duration and temperature significantly affect the water-soluble vitamin contents, especially vitamin C (Klimczak et al., 2007, Romeu-Nadal et al., 2008). Gliguem and BirlouezAragon (2005) reported that low storage temperatures and short storage time are needed to retard vitamin C degradation. This is in agreement with what Rapisarda et al. (2008) reported on the effect of cold storage $\left(6 \pm 1{ }^{\circ} \mathrm{C}, 65 \mathrm{~d}\right)$ on vitamin $\mathrm{C}$. They noted that cold storage positively influenced vitamin $\mathrm{C}$ in blond orange varieties. Reports by Philip et al. (2010) also indicated that retention of most water-soluble vitamins in low temperatures of $-60{ }^{\circ} \mathrm{C}$ showed stable vitamin $\mathrm{C}$ concentration in Clementine and orange juice. The report concluded that careful attention must be given to the reference and the sample protocols handling before analysis else ascorbic acid values may not reflect the concentration in the foods as consumed. Tightly closed solution of working standards mixtures should be stored at room temperature, protected from light and must be used within 36 hrs (Vidovic et al., 2008).

\section{Preparation of Standard Solutions for HPLC}

The stock standard solution is usually weighed in a $100 \mathrm{~mL}$ volumetric flask. Appropriate mass or volume of the standard sample is taken and dissolved in ultra pure water. A buffer such as $1 \mathrm{M}$ Phosphate is sometimes added to bring the $\mathrm{pH}$ to a desirable level (Ciulu et al., 2011; Leibiedzinska et al., 2007). The various components may be mixed together before finally topping up to the $100 \mathrm{~mL}$ mark. Due to the instability of most WSV under light, temperatures, the standard solution must be kept in the dark at $4{ }^{\circ} \mathrm{C}$ and as much as possible prepared daily (Klejdus et al., 2004; Ciulu et al., 2011). Li and Xu (2008) reported that the dried material should be made into powder, mixed with distilled water, and allowed to stand for 10 to 15 minutes at room temperature for the complete homogenization of the water-soluble vitamins into the solution. Vidovic et al. (2008) prepared the stock solution by the dissolution of $\mathrm{B}$ vitamins and test samples in $0.1 \%$ ortho-phosphoric acid at a temperature near boiling for the preparation of multivitamins tablets and syrups prior to HPLC analysis. The working standards were in the concentration range of $0.96-3.00 \mathrm{mg} / \mathrm{mL}$ for vitamin $\mathrm{C}$, $19-53 \mu \mathrm{g} / \mathrm{mL}$ for vitamin $\mathrm{B} 1,8-24 \mu \mathrm{g} / \mathrm{mL}$ for vitamin $\mathrm{B} 2,6-18 \mu \mathrm{g} / \mathrm{mL}$ for vitamin $\mathrm{B} 6,120-360 \mu \mathrm{g} / \mathrm{mL}$ for vitamin B3, and $50-150 \mu \mathrm{g} / \mathrm{mL}$ for vitamin B5. Ghasemi and Abbasi (2005) prepared the stock solution of each vitamin by dissolving $50 \mu \mathrm{g}$ of the vitamin in a $50 \mathrm{~mL}$ volumetric flask and diluted with water to the mark. The working mixtures were made from stock solutions at random in a concentration range of $4-22,1-20,6-26$, and $4-20 \mu \mathrm{g} / \mathrm{mL}$ for vitamins B6, B2, B1 and B0, respectively.

\section{Preparation of test sample for HPLC}

Mostly, preparations of test sample for WSV analysis are done either by mere dissolution of the solute in ultra pure water or by stirrers (handheld, magnetic, ultrasonic baths, etc.) (Ghasemi and Abbasi, 2005; Chen et al., 2006; Ciulu et al., 2011). Depending on the nature and form of the food material, a mere 
dissolution might not be applicable when the test sample cannot be dissolved in its raw form (e.g. dried fruit). In the case of dried tropical fruits or any dried material it is first ground into fine powder, passed through a series of meshes to make sure the particle sizes are uniform prior to dissolution. Other researchers homogenize for approximately $2 \mathrm{~min}$ with blenders and available homogenizers in water, followed by centrifuging, and then filtration as have been stated in preparation standards solutions. Extractant properties that affect the destruction of vitamins once extracted include dissolved oxygen, ionic strength, concentration and type of catalytic trace metals, and the presence of destructive solutes such as chlorine or protective like certain reducing agents. Hence homogenizing should be done in ultraclean vessels free from these destructive Extractant (Owen, 1996)

\section{Simultaneous Determination of Water-Soluble Vitamins}

The water-soluble vitamins are usually ascorbic acid (vitamin C), thiamine (vitamin B1), riboflavin (vitamin B2), niacin (vitamin B3), panthothenic acid (vitamin B5), pyridoxine (vitamin B6), cyanocobalamine (vitamin B12), folic acid, and biotin (Owen, 1996). Apart from vitamin C, the rest is usually termed B-group vitamins. Simultaneous determination of some WSV mostly started in the early 1980s (Kamman et al., 1980; Bama and Dworschiik, 1994). Different instrumental methods were developed by many researchers for use for the simultaneous determination of the water solublevitamins, especially the B-group vitamins. These include electrochemical method (Zhao et al., 1996), spectrophotometry (Ortega-Barales et al., 1998; Zeng et al., 1996), derivative UV spectrophotometry (Morelli, 1994/1995; Morelli and Fresenius, 1996; Shi et al., 1991), spectrofluorimetry (Gong et al., 1994; Liu et al., 1998; Jiang et al., 1997), normal phase and reversed phase TLC (Navas et al., 1993; Zang and Ma, 1989; Bhushan and Parshad, 1994) and HPLC (Amin and Reusch, 1987; El-Gizawy et al., 1991; Zhao et al., 1993; Markopoulou et al., 2002; Ivanovic et al., 1999) as well as capillary electrophoresis ( $\mathrm{Li}$ and $\mathrm{He}, 1997$; Dinelli and Bonetti, 1994). Amin and Reusch (1987) developed a simple, quick, specific and sensitive HPLC method for the determination of four of the B vitamins in a single run in the presence of each other, enabling vitamins to be investigated in the nanogram range as required for pharmacokinetics and bioavailability studies. Barna and Dworschak (1994) assayed vitamins $\mathrm{B} 1$ and $\mathrm{B} 2$ in meat and the liver by HPLC using phosphate buffer ( $\mathrm{pH}$ 3.0) - acetonitrile (84:16, $\mathrm{v} / \mathrm{v})$ containing $5 \mathrm{mM}$ sodium heptasulphonate. A different mobile phase mix of acetonitrile (85:15, $\mathrm{v} / \mathrm{v})$ was used by them for the B1 and B2 determination in liver in the UV-vis detector at 254 $\mathrm{nm}$. These kinds of mix have been reported to be common in situations where the amounts of various components other than the WSV are likely to influence the effectiveness of the separation system. Therefore, the type of food sample has to be emphasized prior to using any of the developed HPLC methods for vitamins analysis.

International bodies such as the International Federation of Fruits Juice Producers (IFFJP) have suggested the HPLC to its members for the determination of vitamin C (IFFJP, 1985). This primarily is because researchers believe that the coupling of chromatographic separation with spectrophotometric, fluorometric, or electrochemical detection makes HPLC analysis far more specific than traditional redox methods. Reports that HPLC permits simultaneous determination of most watersoluble vitamins and their different forms have been documented (Arya et al., 2000). Uncertainties still remain regarding the agreed HPLC method for the determination of vitamin $B_{12}$ since researchers say HPLC is unsuitable for vitamin B12 analysis in food because of their low concentration typically found (Ellenbogen and Cooper, 1992). In the case of biotin, several HPLC methods have been developed but their applicability to foods has not been fully established (Owen, 1996). Other chromatographic systems separate vitamins B1, B6 and B12 when they are present in the same concentration range (Amin and Reusch, 1987) but are hampered when the amount of vitamins B1 and B6 is exceeded by a hundred or even a thousand fold the amount of B12 present in the complex. The determinations of B-complex mainly in tablets using HPLC methods have been extensively described. The most widely used methods for the determination of B-group vitamins are reversedphase HPLC, using a $\mathrm{C}_{18}$ column and aqueousorganic mobile phases, in acidic media. The methods reported in the literature are unable to determine simultaneously all the water-soluble vitamins. Ivanovic et al. (1999) developed and validated a method to assay some water soluble vitamins (B1, B2, B3, B6, C) in solution dosage forms. The recent literature attaches importance to those dosage forms which contain folic acid and vitamin B12, important for the treatment of anaemia, especially in pregnancy. A method based on gas chromatography-mass spectrometry has been reported, but extensive sample preparation is a disadvantage of the procedure (Deutsch and Kolhouse, 1993). Determination of water-soluble vitamins by Liquid Chromatography 
with electrochemical detection has been reported by many researchers (Iwase et al., 1998; Hou and Wang, 1990) for analysis of aseptically packed orange juice, soft drinks, vegetables, multi-component elemental diet, ground meat, processed foods, beer and beverages, and blood plasma. Though electrochemical detection offers advantages of low detection limits with high sensitivity, the oxidation product of some water-soluble vitamins (e.g. vitamin C) cannot be detected in the oxidative mode. Within this period, numerous HPLC methods with fluorescence and UV detections (Eitenmiller et al., 1999; Krautler et al., 1998; Muhamad et al., 1993; Esteve et al., 1998), the capillary electrophoresis (CE) (Vina et al., 1996) and microbiological techniques (Eitenmiller et al., 1999; AOAC, 2003). Interests increased during this period in the simultaneous determination of WSV. For the analysis of fortified foods Albala-Hurtado et al. (1997) have reported the simultaneous determination of vitamins $\mathrm{B}_{1}, \quad \mathrm{~B}_{2}, \quad \mathrm{~B}_{6}, \quad$ nicotinamide, folic acid, and cyanocobalamine using HPLC method with UV detector set at dual wavelengths.

In the early $20^{\text {th }}$ century, many reports have been published with modification and improvement over the previous years. HPLC methods with the capillary electrophoresis were modified by other researchers to elute more of the water-soluble vitamins for different type of food materials (Gatti and Giola, 2005; Indyk et al., 2002). In many of the published HPLC procedures, researchers were mainly interested in finding methods that will elute the target watersoluble vitamins within the shortest possible time in order to decrease significantly the overall chromatography run time. Sharpless et al. (2000) reported a standard reference material for the determination of vitamins in the food-matrix but employed several methods including the HPLC and other traditional methods. The use of different methods of all the vitamins in his method is a disincentive to its usage for routine analysis. Klejdus et al. (2004) developed an HPLC method capable of eluting both water-soluble and fatsoluble vitamins in a single run using a combined isocratic and linear gradient elution with $0.01 \%$ trifluroacetic acid ( $\mathrm{pH} 3.9$ ) and methanol as mobile phase at the flow rate of $0.7 \mathrm{~mL} / \mathrm{min}$. In their report, it was concluded that the most suitable wavelength for simultaneous determination of both watersoluble and fat-soluble vitamins is $280 \mathrm{~nm}$ and the method is applicable to solid sample of pharmaceutical preparation (B-complex, fortified powdered drinks (multi-vitamins) and food sample. Even though the method proposed by Klejdus et al. (2004) covers simultaneously a wide range of both the water-soluble and fat-soluble vitamins, the coupling of the HPLC to the quadruple mass spectrometer operated in the positive ESI mode made the experimental set-up expensive and therefore a major disadvantage for it adoption.

Reports show that the simultaneous determination of several water-soluble vitamins is difficult and often many different analyses have to be performed (Amidzic et al., 2005). Besides, Amidzic et al. (2005) reported a simple reversed phase HPLC method for the simultaneous determination of vitamins $\mathrm{B}_{1}, \mathrm{~B}_{3}, \mathrm{~B}_{6}, \mathrm{~B}_{12}$, and folic acid, in multivitamin tablets; but their method precludes vitamin C. Both dual detection wavelengths and temperatures of $290 \mathrm{~nm} / 35^{\circ} \mathrm{C}$ and $550 \mathrm{~nm} / 25{ }^{\circ} \mathrm{C}$ were used for the simultaneous detection of vitamins $\mathrm{B}_{1}, \mathrm{~B}_{2}, \mathrm{~B}_{6}$, folic acid and $\mathrm{B}_{12}$ respectively. This may have been the complexities surrounding the elution strengths of individual WSV. Heudi et al. (2005) have reported liquid chromatography separation of nine water-soluble vitamins with LC-MS analysis in premixes. The HPLC procedures with the appropriate detection system are considered as the most convenient methods for the determination of WSV especially the B-group (Lebiedzinska et al., 2007). The HPLC coupled with electrochemical detector (HPLC-ED) is believed to be sensitive and selective method for the determination of redox compounds. It is one of the analytical methods that has been recommended for the determination of B-group vitamins in pharmaceutical formulations and biological matrices (Ekinci, 2005; Marszall et al., 2005; Mimica et al., 2002).

For analysis of seafood (fish, mussels, oysters) and food products of plant origin (apple juices and fortified fruit juices), Lebiedzinska et al. (2007) have reported an HPLC method with ED and UV detection for the simultaneous determination of vitamins B1, B6, and B12. The method offers high accuracy, better repeatability and reproducibility and a relative short time for quantification of the three water-soluble vitamins assayed in food samples. The simple mobile phase and isocratic elution used to separate the thiamine, pyridoxine, pyrioxal, pyridoxamine, and vitamins B12 yielded low detection limits, good sensitivity and resolution with a minimum analysis time of $17 \mathrm{~min}$. However, the combination of acid digestion and enzymatic extraction to release proteinbound and phosphorylated vitamins prior to HPLC made the preparation of samples long. The relatively long time used for the preparation of the food products of plant origin alone (over $42 \mathrm{hrs}$ ) makes this method unsuitable for rapid routine analysis. Ghasemi and Abbasi (2005) proposed a simple and rapid simultaneous spectrophotometric method using the 
parallel factor analysis (PARAFAC) for four of the group B complex vitamins (folic acid, B1, B2, and B6). However, their detection wavelength for the PARAFAC model was in the range of $400-600 \mathrm{~nm}$, which preclude simultaneous determination involving ascorbic acid, because vitamin C elute strongly UV light at $245 \mathrm{~nm}$. Also, even though the method proposed is simple and suitable for routine analysis, the precision and accuracy of such a method may not be reliable. In 2008 Vidovic et al. reported simultaneous determination of some water-soluble vitamins using the HPLC. Their method is simple and covers a wide range of the water-soluble vitamins (vitamin C, B1, B2, B3, B5, and B6). However, the method did not cover the other water-soluble vitamins such as folic acid, biotin, and vitamin $\mathrm{B}_{12}$.

In recent times, highly sensitive and rapid method has been developed by Ciulu et al. (2011) for the simultaneous determination of water-soluble vitamins (B2, B3, B5, folic acid, and C) in honey by LC with UV-vis detection. In their method all the vitamins were eluted in a single run within $17 \mathrm{~min}$. This seem to be a major breakthrough in the quantification of simultaneous determination of vitamins in honey, but not for other food materials since this elution time for all five WSV have been reported in 2008 by Vidovic et al. Currently, research is ongoing and many more HPLC procedures for simultaneous determination of all the WSV in a single run are yet to be documented.

\section{Chromatographic conditions for determination of water-soluble vitamins}

Liquid chromatography (LC) has commonly been used for the separation of water-soluble vitamins, thus resulting in a number of such methods. Several LC procedures using highly polar columns and mobile phases consisting of various proportions of acetonitrile and methanol in the presence of aqueous solution of $5 \mathrm{mM}$ heptafluorobutyric acid under gradient elution mode have been used (Chen et al., 2006). Simultaneous water-soluble vitamins have been determined in different samples by LC, using $0.1 \%$ trifluoroacetic acid-sodium dihydrogen phosphate (Chen et al., 2006), methanol-phosphate buffer (10:90) and $0.018 \mathrm{M}$ trimethylamine, $\mathrm{pH} 3.55$ (Lebiedzinska et al., 2007), $0.05 \mathrm{M}$ ammonium acetate- methanol (Moreno \& Salvado, 2000), acetonitrile-trifluoroacetic acid $(0.025 \%$, v/v) (Ciulu et al., 2011), acetonitrile-0.0125M hexane-1-sulfonic acid sodium salt (in $0.1 \%$ phosphoric acid buffer, $\mathrm{pH}$ 2.4 (Vidovic et al., 2008), methanol - $5 \mathrm{mM}$ heptane1 -sulphonic acid sodium salt $/ 0.1 \%$ triethylamine TEA (25:75 v/v)(Amidzic et al., 2005) as mobile phases. Many researchers have monitored of the UV absorbance at 210 and 254 in gradient elution of the mobile phase (85:15). However Moreno and Salvado (2000) monitored the WSV at $270 \mathrm{~nm}$ with the exception of vitamin B12 where $362 \mathrm{~nm}$ was used. A liquid chromatography with dual wavelength detector is widespread in most HPLC experiments for the simultaneous determination of WSV. This may be due to the reason that some WSV elute strongly UV light at some specific wavelength than others. A typical example is vitamins $\mathrm{C}$, which has been observed to strongly elute UV light at $254 \mathrm{~nm}$ but weakly at $210 \mathrm{~nm}$. Highly sensitive and rapid methods have been proposed for the simultaneous determination of water-soluble vitamins in honey, urine, nutritional supplements, and food samples by LC with UV-vis detection (Ciulu et al., 2011; Vidovic et al., 2008; Chen et al., 2006). Detection of target components was done using electrospray ionization mass spectroscopy (ESI-MS) switching continuously from positive ion mode to negative ion mode. Whilst some researchers used internal methods to quantify WSV after separation, the majority of the researchers used internal standards based on peaks to quantify concentration. Typically, Huppuric acid (Chen et al., 2006) and external method based on the peak areas of the eluted WSV (Vidovic et al., 2008) are used as the internal standard for quantification. The method proposed by Ciulu et al. (2011) is rapid; in that, all five analytes were completely eluted within $17 \mathrm{~min}$ and the whole chromatographic run was completed in $22 \mathrm{~min}$. However, the method proposed was not validated for vitamins B1, B6, B12 and biotin. It is also common to see that some researchers used different mobile phases for simultaneous determination of the B-group vitamins. For example, Amidzic et al. (2005) used methanol$5 \mathrm{mM}$ heptanesulphonic acid sodium salt / $0.1 \%$ triethylamine TEA $(25: 75 \mathrm{v} / \mathrm{v})$ [the $\mathrm{pH} 2.8$ was adjusted with orthophosphoric acid] for simultaneous determination of vitamins B1, B3, B6, and folic acid but used methanol-water $(22: 78 \mathrm{v} / \mathrm{v})$ as a second mobile phase. Available literature showed that flow rates between 0.7 and $2 \mathrm{~min} / \mathrm{L}$ are used by many researchers, with the $0.2-0.45 \mu \mathrm{m}$ filter used to filter all mobile phases and degassed with an ultrasonic bath. The presence of citrate is reportedly responsible for causing voids in the column, thus preventive measures are needed while analyzing citrus juices and samples of high acidic contents. In addition, the use of an acidic mobile phase might affect the columns life of columns packed with silica as silicate is dissolved slowly by such solutions. Though acetonitrile has most commonly been used as a mobile phase, handling of this solvent requires precaution to avoid breathing its vapour (Arya et al., 2000). 


\section{Optimization of Chromatographic method}

Reports indicate that even excellent conditions on the reversed phase high performance liquid chromatography RP-HPLC for the determination of most important WSV might not be applicable to all sample materials. A typical example is that WSV in premises used for food fortification present a different complexity compared with WSV in honey, urine, dried fruits and vegetables (Ciulu et al., 2011). Due to the fact that the complexities surrounding specific materials are different, a careful optimization of the HPLC procedure is suggested. The optimization work mainly addresses the program of the chromatographic elution and the proper choice of the UV wavelength in order to maximize both resolution and sensitivity.

\section{Application to Drying of fruits}

Water soluble vitamins are highly soluble in aqueous solutions and are unstable. This show that potential exist for significant losses by leaching from freshly cut or bruised surfaces of fruits. Chemical degradation exists for some of these vitamins. For example vitamin $\mathrm{C}$ can be oxidized to dehydroascorbic acid (DHAA), followed by hydrolysis to 2, 3-diketogulonic acid and further oxidation, dehydration, and polymerization to form a wide array of other nutritional inactive products (Hernandez et al., 2006).

These indicate that bruised-free fruits should be selected for drying. Since most drying research involves chemical pretreatments, blanching, and osmotic dehydration, a minimum soaking time is recommended to avoid excessive leaching of watersoluble vitamins prior to drying. Also, in the storage of the dried products, care should be taken to prevent oxidation of the vitamins by sealing them in well packaged air-tight containers.

\section{Conclusions}

In the use of HPLC for the determination of WSVs, it should be noted that WSVs are usually unstable and therefore the reference and sample protocols must be handled with great care. Separation and quantification should be done with a high level of precision and suitable methods. HPLC method for analyzing one or two of the water-soluble vitamins are common but a single run HPLC method for simultaneous analysis for all the water-soluble vitamins are undocumented. In the review, the shortest elution time reported for simultaneous assay of at least five of the water-soluble vitamins was $17 \mathrm{~min}$. It is therefore important that we have a single most sensitive HPLC method, which is robust, rapid and efficient for determining all the water-soluble vitamins in a single run.

\section{Aknowledgements}

The authors wish to extend their appreciation to the Open Fund of Jiangsu Provincial Key Laboratory for Physical Processing of Agricultural Products (JAPP2010-6), the Jiangsu Province Science and Technology Support Project (BE2011382), the Jiangsu University Graduate Research Innovation Program (CXZZ12_0700), the Jiangsu University Student Scientific Research Project (11A346), the Jiangsu University Student Practice Innovation Training Program, and the Priority Academic Program Development (PAPD) of Jiangsu Higher Education Institutions for their financial support toward the study.

\section{References}

Albala-Hurtado, S., Veciana-Nogues, M.T., IzquierdoPulido M., Marine-Font, A. (1997): Determination of water-soluble vitamins in infant powder by high performance liquid chromatography, J. Chromatogr. A 778, 247-253.

Amidzic, R., Brboric J., Cudina O., Vladimirov, S. (2005): RP-HPLC Determination of vitamins B1, B3, B6, folic acid, and B12 in multivitamin tablets, J. Serbia Society 70 (10), 1229-1235.

Amin, M., Reusch, J. (1987): Simultaneous determination of vitamins $\mathrm{B}_{1}, \mathrm{~B}_{2}, \mathrm{~B}_{6}$, and $\mathrm{B}_{12}$ in pharmaceutical preparations, J. Chromatogr. 390 (2), 448-453.

AOAC (2003): Association of Official Analytical Chemists, Washington, DC.

Arya, S.P., Mahajan M., Jain, P. (2000): Nonspectrophotometric methods for the determination of vitamin C, Analyica Chimica Acta 417 (1), 1-14.

Barna, E., Dworschak, E. (1994): Determination of thiamine (vitamin B1) and Riboflavin (vitamin B2) in meat and liver by HPLC, J. Chromatogr. A 668 (2), 359-363.

Chen, Z., Chen, B., Yao, S. (2006): HPLC/EIMS for simultaneous determination taurine and 10 watersoluble vitamins in multivatimin tablets, Analytica Chimical Acta 569, 169-175.

Ciulu, M., Solinas, S., Floris, I., Panzanelli, A., Pilo, M.I, Piu, P.C., Spano, N., Sanna, G. (2011): RP-HPLC determination of water-soluble vitamins in honey, Talanta 83, 924-929.

Deutsch, J.C., Kolhouse, J.F. (1993): Ascorbate and dehydroascorbate measurements in aqueous solutions and plasma determined by gas chromatography-mass spectrometry, Anal. Chem. 65 (4), 321-326.

Eitenmiller, R.R., Landen Jr, W.O. (1999): Vitamin Analysis for the Health and Food Sciences, CRC Press, Boca Raton, FL, 231-505. 
Ekinci, R., Kadakal, C. (2005): Determination of seven water soluble vitamins in Tarhana, a traditional Turkish cereal food, by high performance liquid chromatography, Acta Chromatogr. 15, 289-297.

Esteve, M.J., Farre, R., Frigola, A., Garcia-Cantabella, J.M. (1998): Determination of vitamin B6 (pyridoxamine, pyridoxal, and pyridoxine) in pork meat and pork meat products by liquid chromatography, J. Chromatogr. A 795 (2), 383-387.

Gatti, R., Giola, M.G. (2005): Liquid chromatographic determination with fluorescence detection of B6 vitamers and riboflavin in milk and pharmaceuticals, Anal. Chimica Acta 538 (1-2), 135-141

Ghasemi, J., Abbasi, B. (2005): Simultaneous spectrophotometric determination of group B vitamins using parallel factor analysis: PARAFAC, Journal of Chinese Chemical Society 52, 1123-1129.

Gliguem, H., Birlouez-Aragon, I. (2005): Effects of sterilization, packaging, and storage on vitamin $\mathrm{C}$ degradation, Protein Denaturation, and Glycation in Fortified Milks, J. Dairy Sci. 88 (3), pp 891-899.

Hernandez, Y., Gloria Lobo, M., Gonzalez, M. (2006): Determination of vitamin $\mathrm{C}$ in tropical fruits: $\mathrm{A}$ comparative evaluation of methods. Analytical, Nutritional, and Clinical Methods, Food Chem. 96 (4), 654-664.

Heudi, O., Kiline, T., Fontannaz, P. (2005): Separation of water-soluble vitamins by reverse-phase high performance liquid chromatography with ultra-violet detection: application to polyvitamined premixes, $J$. Chromatogr. A 1070, 49-56.

Indyk, H., Persson, B., Caselunghe, M., Filouzi, E., Wollard, D. (2002): Determination of vitamin B12 in milk products and selected foods by optical biosensor protein binding assay: method comparison, J. AOAC Intl. 85, 72-82.

Inga, K., Malecka, M., Szlachta, M., Gliszcynska-Swiglo A. (2007): Effect of storage on the content of polyphenols, vitamin $\mathrm{C}$ and the antioxidant activity of orange juices, J. Food Compos. Analysis 20 (3-4), pp 313-322.

Kamman, J.F., Labuza, T.P., Warthesen, J.J. (1980): Thiamin and riboflavin analysis by high performance liquid chromatography, J. Food. Sci. 45 (6), 14971499.

Klejdus, B., Petrlová, J., Potešil, D., Vojťech, A., Mikelová R., Vacek J., Kizek R., Kubánn, V. (2004): Simultaneous determination of water- and fat-soluble vitamins in pharmaceutical preparations by highperformance liquid chromatography coupled with diode array detection, Anal. Chimica Acta 520, 57-67.

Krautler, B., Arigoni, D., Golding, B.T. (1998): Vitamin $\mathrm{B}_{12}$ and $\mathrm{B}_{12}$ proteins, John Wiley \& Sons-VCH, Weinheim.

Lebiedzinska, A., Marszall, M.L., Kuta, J., Szefer, P. (2007): RP-HPLC method with coulometric electrochemical and ultraviolet detection for the quantification of Vitamins B1, B6 and B12 in animal and plant foods, J. Chromatogr. A 1173 (1-2), 71-80.
Leskova, E., Kubikova, J., Kovacikova, E., Kosicka, M., Porubska, J., Holcikova, K. (2006): Vitamin losses: retention during heat treatment and continual changes expressed by mathematical models, J. Food Compos. Analysis 19 (4), 252-276.

Li, Y., Xu, S. (2008): Preparation of Garlic Powder with high allicin content, Agric. Sci. China 6 (7), 890-898.

Marszall, M.L., Lebiedzinska, A., Czarnowski, W., Szefer, P. (2005): Highe-performance liquid chromatography method for the simultaneous determination of thiamine hydrochloride, pyridoxine hydrochloride and cyanocobalamin in pharmaceutical formations using coulometric electrochemical and ultraviolet detection, J. Chromatogr. A 1094 (1-2), 91-98.

Mimica, D., Bedioui, F., Zagal, J.H. (2002): Reversibility of the L-cysteine/L-cystine redox process at physiological $\mathrm{pH}$ on graphite electrodes modified with coenzyme B12 and vitamin B12, Electrochimica Acta 48, 323-329.

Muhammad, K., Briggs, D., Jones, G. (1993): The appropriateness of using cyanocobalamin as calibration standards in Lactobacillus leichmannii A.T.C.C. 7830 assay of vitamin $\mathrm{B}_{12}$, Food Chem. 48 (4), 427-429.

National Institute of Standard and Technology. Standard Reference Materials. [Accessed 21 July 2014]. http://ts.nist.gov/MeasurementServices/ReferenceMat erials/232.cfm.

Owen, R., Fennema (1996): Food Chemistry. Third edition. Marcel Dekker, Inc. New York, USA, 531-610.

Paolo, R., Bianco, M.L., Pannuzzo, P., Timpanro, N. (2008): Effect of cold storage on vitamin C, phenolics and antioxidant activity of five organe genotypes, J. Postharvest Biol. Technol. 49 (3), 348354.

Philips, K.M., Terrago-Trani, M.T., Gebhardt, S.E., Exler, J., Patterson, K.Y., Haytowitz, D.B., Pehrsson, P.R., Holden, J.M. (2010): Stability of Vitamin C in Frozen raw fruit and vegetable homogenates, J. Food Compos. Analysis 23 (3), 253-259.

Sharpless, K.E., Margolis, S., Thomas, J.B. (2000): Determination of vitamins in food-matrix standard reference materials, J. Chromatogr A. 881, 171-181.

Vidovic, S., Stojanovic, B., Veljkovi, J., Prǎzic-Arsic, L., Roglic, G., Manojlovic D. (2008): Simultaneous determination of some water-soluble vitamins and preservatives in multivitamin syrup by validated stabilityindicating high-performance liquid chromatography method, J. Chromatogr. A 1202, 155-162.

Revanasiddappa, H. D., Veena, M. A. (2008): Sensitive Spectrophotometric Methods for the Determination of Ascorbic Acid, E-J. Chem. 5 (1), 10-15.

Romeu-Nadal, M., Casetellote, A.I., Lopez-Sabater, M.C. (2008): Effect of cold storage on vitamins C and E and Fatty acids in human milk, Food Chem. 106 (1), 65-70.

Received: April 10, 2014

Accepted: August 25, 2014 